\title{
Mastering Energy Management During Rowing Using Virtual Reality
}

\author{
Charles P. Hoffmann ${ }^{*}$ Alessandro Filippeschi ${ }^{* \dagger}$ Emanuele Ruffaldi $^{* \dagger}$ \\ Sébastine Blanc ${ }^{*}$ Luc Verbrugge ${ }^{*}$ Benoît G. Bardy ${ }^{*}$ \\ (*) Movement to Health (M2H) Laboratory, EuroMov, Montpellier-1 University, France \\ (*) Scuola Superiore Sant'Anna, Pisa, Italy \\ E-mail: charles.hoffmann@univ-montp1.fr
}

\begin{abstract}
This study focused on one of the most important factors in rowing performance which is the ability demonstrated by elite rowers to regulate their effort during a $2000 m$ race in order to produce their best performance. Experts are known to adopt a particular pacing strategy with a fast-start profile. The purpose of our study was to test by using virtual reality whether novice rowers were able to acquire this energy management skill during a $2000 \mathrm{~m}$ race, with positive consequences for their rowing performance. Participants from the Avatar group were instructed to track a virtual boat, which velocity was previously calibrated to follow the appropriate to-be-learned velocity profile. This group was contrasted with a control group, which followed a classic training procedure. Our results indicate that the avatar group improved its performance (decrease in race duration), learned and maintained the expert profile. These effects were absent in the control group. Together, these results indicate that virtual reality can be used to accelerate the learning of energy-related skills.
\end{abstract}

\section{Introduction}

Performance in rowing, e.g., race duration over a particular distance, is determined by several factors, which should be taken into account during training. In our research, we focused on one important factor, the ability demonstrated by elite rowers to manage their energy stocks during a $2000 \mathrm{~m}$ race. Several authors have shown that experts adopt a particular pacing strategy. Their velocity corresponds to a fast-start profile, with the first $500 \mathrm{~m}$ performed at $103.3 \%$ of the average whole race speed, and with the subsequent sectors rowed at $99.0 \%, 98.3 \%$ and $99.7 \%$ of the average speed, respectively [1].

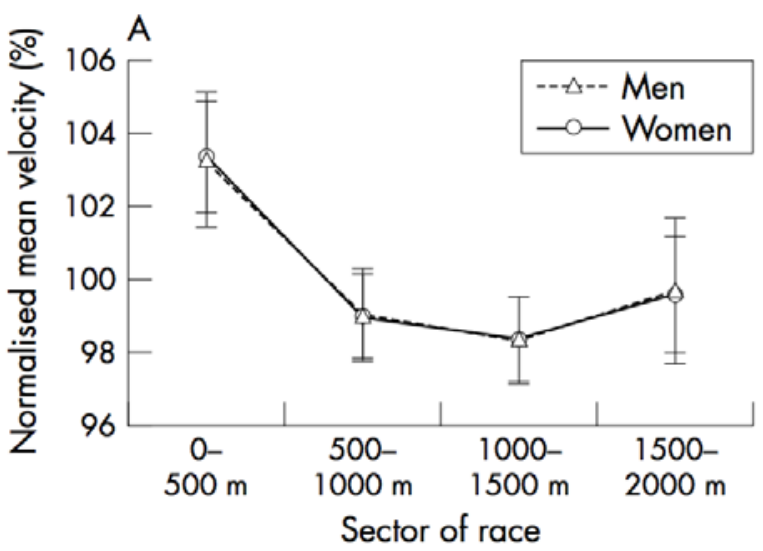

Fig 1. Velocity profile of expert rowers obtained by Garland (2005) corresponding to best performance

The purpose of our study was to test by using virtual reality whether novice rowers were able to acquire this energy management skill during a $2000 \mathrm{~m}$ race, with positive consequences for rowing performance and retention. A better management of energy consumption after training, for the same training workload, was expected for the avatar group compared to a control group.

\section{Method}

\subsection{Subjects}

15 males novice rowers whose ages ranged between 21 and 29 years completed the protocol. They all signed an informed consent before participating in the experiment, which was approved by the regional ethic review board (Comité Consultatif de Protection des Personnes de Nîmes Sud-Méditerranée 3).

This is an Open Access article distributed under the terms of the Creative Commons Attribution-Noncommercial License 3.0, which permits unrestricted use, distribution, and reproduction in any noncommercial medium, provided the original work is properly cited. 


\subsection{Procedure}

Participants were divided into two groups. The control group $(n=8)$ followed a classic indoor rowing training which consisted of several $2000 \mathrm{~m}$ races. The avatar group $(n=7)$ benefitted from an energymanagement information - represented by an avatar boat - visible on a large screen located in front of participants operating a Concept 2 indoor rowing machine. Participants (of the avatar group) were instructed to track the virtual boat, which velocity was previously (individually) calibrated to follow the appropriate to-be-learned velocity profile along the $2000 \mathrm{~m}$ race (see Fig 2). The virtual boat was gradually removed during the training session. Our protocol followed a pre/post/retention test design. We compared pre- and post-test to evaluate the effect of the velocity profile accelerator. The retention test was performed in order to check if the learned-velocity-profile was maintained after a period of 30 days. The two groups performed the same number of trials, and overall, the same quantity of practice.

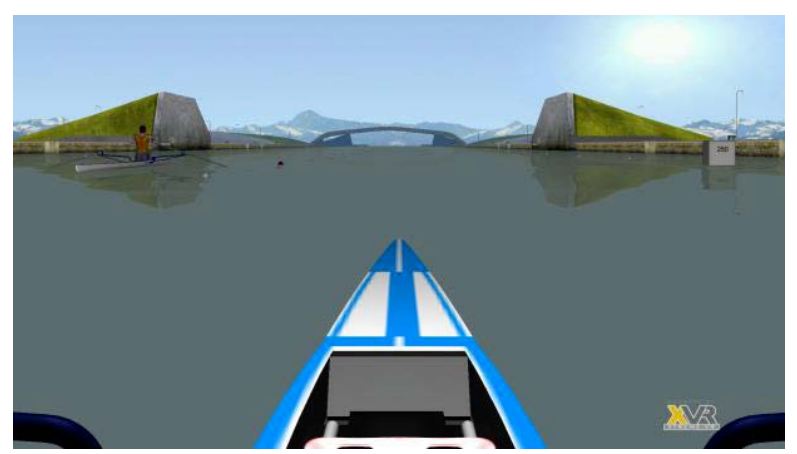

Fig 2. Representation of the avatar boat

\section{Results / Discussion}

A two-way repeated measurement ANOVA was performed to assess the evolution of performance in term of race duration. Our results indicate that the avatar group improved its performance against the control group in the post-test (Fig 3.) $\left(\mathrm{F}_{2,12}=4,1471\right.$; $\mathrm{p}<0.05)$ and maintained this advantage during the retention-test. In a qualitative manner, we can see that the avatar group reproduced more accurately the expert profile than the control group during the post-test (Fig 5A.) and the retention test (Fig 5B.). However the difference between the two groups failed to reach significance. Concerning the oxygen consumption, we found no differences for the avatar group between pre/post/retention tests. Because participants from this group completed the $2000 \mathrm{~m}$ race faster than the control group with no increase in $\mathrm{O}_{2}$ consumption, they were thus more efficient.

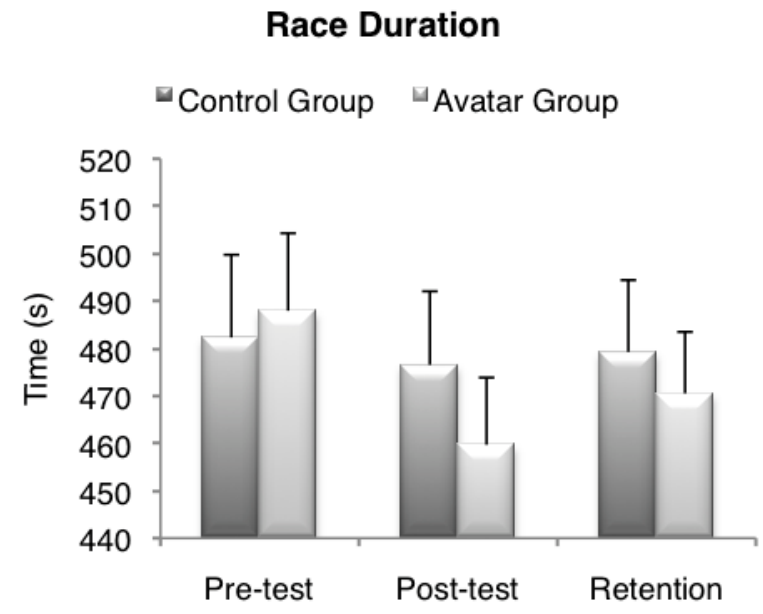

Fig 3. Race duration for the avatar group (black) and the control group (grey) in pre, post, and retention sessions

\section{Conclusion}

Our results indicate that virtual reality can be used to accelerate the learning of energy-related skills, in a relatively short period of time (4-5 weeks). In addition, this learning lead to better performance in term of race duration. These results open up important issues in VR training concerning the transferability of this pacing strategy to other sports with races of similar duration (6-8 minutes), such as running or sprint cycling for instance.

\section{References}

[1] S.W. Garland, An analysis of the pacing strategy adopted by elite competitors in $2000 \mathrm{~m}$ rowing, British Journal of Sports Medicine, pages. 39-42, 2005 


\section{A. Race Strategy Post-Test}

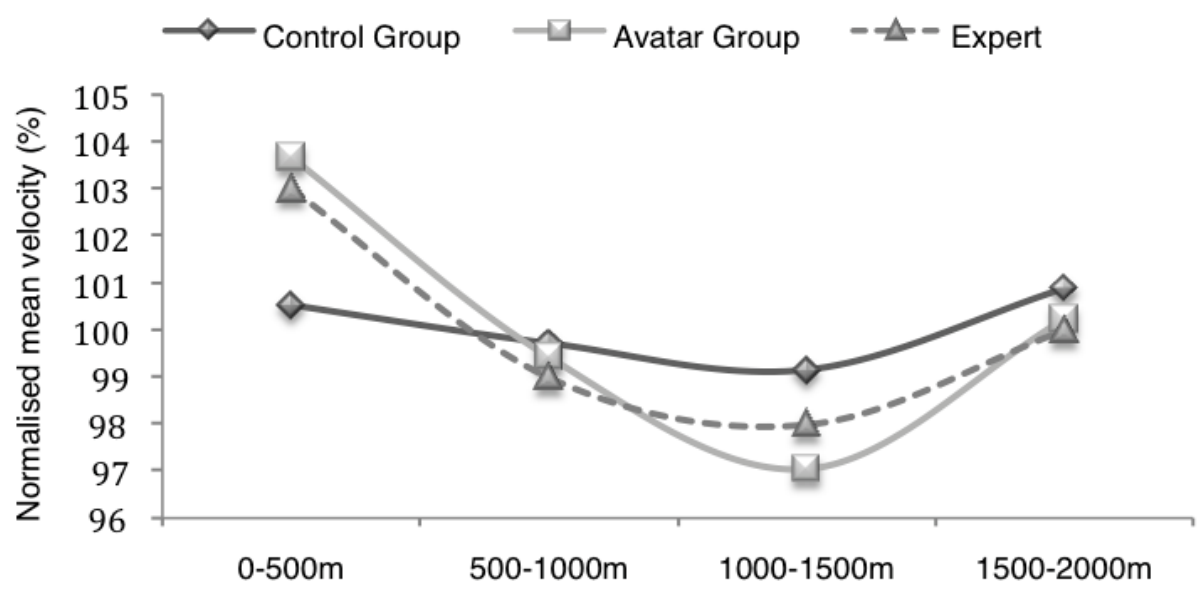

\section{B. Race Strategy Retention}
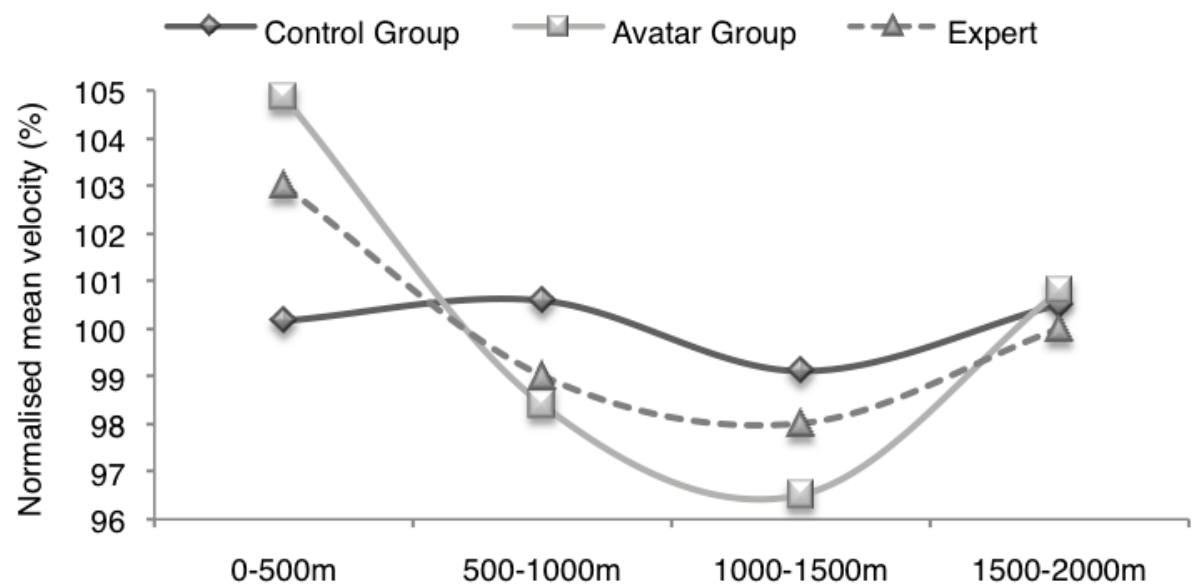

Fig 4: (A). Race pace profiles comparing post-test for the control group (straight black line), for the avatar group (grey line) and the expert profile (dashed black line). (B). Race pace profiles comparing retention-test for the control group (straight black line), for the avatar group (grey line) and the expert profile (dashed black line). 